\title{
A Free Terminal Ileal Perforation as the Presenting Sign of Crohn's Disease in an Adolescent Female
}

\author{
Zlatan Zvizdic ${ }^{1}$, Emir Milisic ${ }^{1}$, Faruk Skenderi ${ }^{2}$, Irmina Sefic Pasic ${ }^{3}$, Semir Vranic ${ }^{4}$ \\ ${ }^{1}$ Clinic of Pediatric Surgery, University Clinical Center Sarajevo, Sarajevo, Bosnia and Herzegovina, ${ }^{2}$ Department of Pathology, \\ University Clinical Center Sarajevo, Sarajevo, Bosnia and Herzegovina, ${ }^{3}$ Department of Radiology, University Clinical Center \\ Sarajevo, Sarajevo, Bosnia and Herzegovina, ${ }^{4}$ College of Medicine, QU Health, Qatar University, Doha, Qatar
}

Correspondence: semir.vranic@gmail.com; Tel.: + 9744403 7873; Fax.: + 97444033344

Received: January 5, 2020; Accepted: January 27, 2020

Key Words: Free Intestinal Perforation • Diffuse Peritonitis • Crohn's Disease • Children • Surgery.

A previously healthy 14-year-old girl was referred to our hospital for management of an acute abdomen. She had a previous history of colicky abdominal pain with distension, diarrhea, and vomiting lasting over the past three days. On examination, she was exhausted, dehydrated and pale. BP was $105 / 70 \mathrm{~mm} \mathrm{Hg}$ and pulse rate was 130 per minute. The abdomen was distended and warm, with diffuse abdominal tenderness. An erect x-ray abdomen was taken revealing a free gas collection under the diaphragm with dilated bowel loops. CT scan confirmed the presence of pneumoperitoneum and intra-abdominal fluid around the spleen and liver (Fig. 1A-B). After adequate preoperative preparation and intravenous administration of broad-spectrum antibiotics, the patient underwent exploratory laparotomy under general anesthesia. Laparotomy was made through a midline incision and a large amount of turbid free fluid was found within the abdominal cavity. The thickened terminal ileum was covered with the omentum. The omentum was subtotally resected and a single perforation on the antimesenteric side of the terminal ileum was found. The perforation was located approximately $15 \mathrm{~cm}$ proximal to the ileocecal valve (Fig. 1C). The mesentery of the terminal ileum was thickened and friable. The ileum was also extensively altered in a length of about $35 \mathrm{~cm}$ from the ileocecal junction, while it was still about $70 \mathrm{~cm}$ of the ileum with the changes that were highly suggestive to Crohn's disease based on the visual inspection (fat wrapping, inflammatory changes, and wall thickening) and the findings of intestinal palpation (induration, thickening of the mesenteric edge, luminal narrowing). The appendix vermiformis and the cecum also showed signs suggestive of Crohn's disease. Since we opted for a sparing surgical treatment, approximately $35 \mathrm{~cm}$ of the affected segments of the ileum and the cecum, the appendix vermiformis and approximately $20 \mathrm{~cm}$ of the ascending colon were resected (Fig. 1D). The primary hand-sewn endto-end anastomosis in one layer was done. There were no complications during the surgery. Postoperatively, parenteral nutrition was applied and replaced subsequently with oral feeding. The patient had an uneventful postoperative course and was discharged on the ninth day. The histopathologic examination of the resected specimen confirmed that perforation occurred due to Crohn's disease affecting the terminal ileum (Fig. 1E-F). The patient received postoperative prophylaxis (mesalamine) 

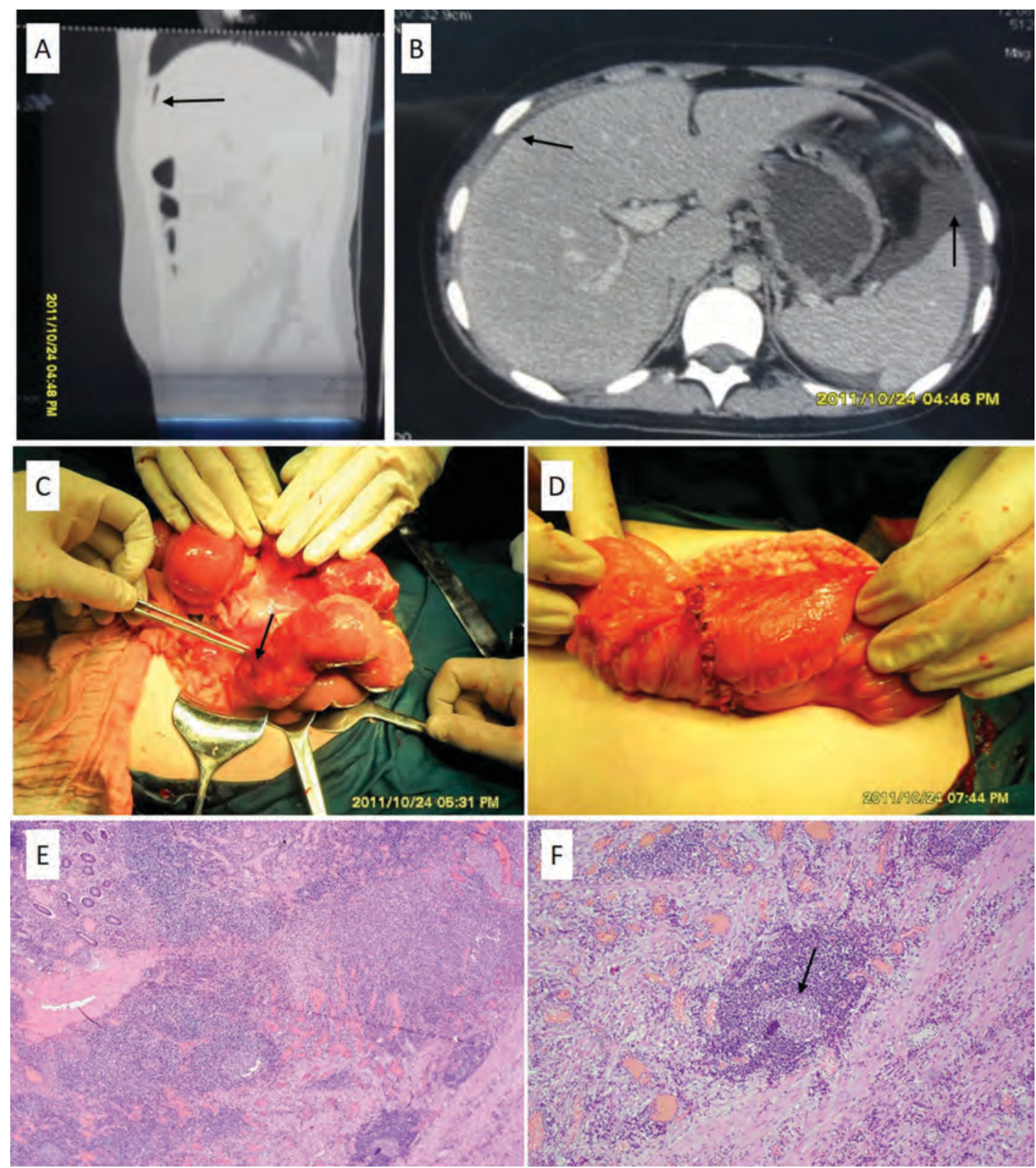

Fig. 1A-F. A-B: Computed tomography scan showed a free gas under the diaphragm (black arrow) (A); Opaque, more dense fluid was found around the spleen and the liver (white arrow) (B); C-D: Intraoperative findings: Site of free ileal perforation (black arrow) (C); Hand-sewn end-to-end ileocolic anastomosis in one layer (D); E-F: Microscopic images showing a transmural propagation of an extensive mixed inflammatory infiltrate composed of neutrophils, eosinophils, macrophages, lymphocytes and plasma cells (E); Occasionally, epithelioid, noncaseous granulomas surrounded by dense lymphocytic infiltrates were seen within the small bowel wall (black arrow) $(F)$.

over a period of two years, is currently well, and remains in remission at an eight-year follow-up.

Authors' Contributions: Conception and design: $\mathrm{ZZ}$ and SV; Acquisition, analysis and interpretation of data: ZZ, EM, FS, ISP, SV; Drafting the article: ZZ and SV; Revising it criti- cally for important intellectual content: ZZ and SV; Approved final version of the manuscript: $\mathrm{ZZ}$ and SV.

Conflict of Interest: The authors declare that they have no conflict of interest. 